Article

\title{
Strain-Specific Adaptations of Streptococcus mitis-oralis to Serial In Vitro Passage in Daptomycin (DAP): Genotypic and Phenotypic Characteristics
}

\author{
Nagendra N. Mishra ${ }^{1,2, *}$, Truc T. Tran ${ }^{3,4}$, Cesar A. Arias ${ }^{3,4}$, Ravin Seepersaud ${ }^{5,6}$, \\ Paul M. Sullam ${ }^{5,6}$ and Arnold S. Bayer ${ }^{1,2}$ \\ 1 Division of Infectious Diseases, The Lundquist Institute at Harbor-UCLA Medical Center, \\ Torrance, CA 90502, USA; abayer@lundquist.org \\ 2 David Geffen School of Medicine at the University of California, Los Angeles (UCLA), CA 90024, USA \\ 3 Center for Antimicrobial Resistance and Microbial Genomics, Division of Infectious Diseases, \\ University of Texas McGovern School of Medicine, Houston, TX 77030, USA; \\ Truc.T.Tran@uth.tmc.edu (T.T.T.); cesar.arias@uth.tmc.edu (C.A.A.) \\ 4 Antimicrobial Resistance Unit and International Center for Microbial Genomics, Universidad El Bosque, \\ Bogota 10121, Colombia \\ 5 University of California, San Francisco, CA 94143, USA; ravin.seepersaud@icloud.com (R.S.); \\ paul.sullam@ucsf.edu (P.M.S.) \\ 6 VA Medical Center, San Francisco, CA 94121, USA \\ * Correspondence: nnmishra@ucla.edu or nmishra@lundquist.org; Tel.: +1-310-222-4013
}

Received: 17 July 2020; Accepted: 13 August 2020; Published: 15 August 2020

check for updates

\begin{abstract}
Viridans group streptococci (VGS), especially the Streptococcus mitis-oralis subgroup, are pivotal pathogens in a variety of invasive endovascular infections, including "toxic shock" in neutropenic cancer patients and infective endocarditis (IE). Previously, we showed that the serial in vitro passage of $S$. mitis-oralis strains in sublethal daptomycin (DAP) resulted in rapid, high-level and stable DAP-resistance (DAP-R), which is accompanied by distinct changes in several genotypic and phenotypic signatures: (1) the disappearance of two key membrane phospholipids, phosphatidylglycerol (PG) and cardiolipin (CL); (2) increased membrane fluidity; (3) increased positive surface charge; (4) single nucleotide polymorphisms (SNPs) in two loci involved in CL biosynthesis ( $p g s A ; c d s A)$; and (5) DAP hyperaccumulation. The current study examined these same metrics following in vitro serial DAP passages of a separate well-characterized $S$. mitis-oralis bloodstream isolate (SF100). Although some metrics seen in prior DAP post-passage strains were recapitulated with SF100 (e.g., pgsA SNPs, enhanced membrane fluidity), we observed the following major differences (comparing the parental versus post-passage variant): (1) no change in PG content; (2) reduced, but not absent, CL, with enhancement in phosphatidic acid (PA) content; (3) an unusual pattern of CL localization; (4) significantly decreased positive surface charge; (5) no difference in DAP accumulation; and (6) no $c d s A$ SNPs. Thus, S. mitis-oralis strains are not "pre-programmed" phenotypically and/or genotypically to adapt in an identical manner during the evolution of the DAP-R.
\end{abstract}

Keywords: Streptococcus mitis-oralis; daptomycin resistance

\section{Introduction}

Invasive infections caused by the $S$. mitis-oralis subgroup of viridans group streptococci (VGS), especially endovascular syndromes, are increasing in both nosocomial and community settings [1-4]. For example, S. mitis-oralis is the second leading cause of infective endocarditis (IE) in the developing world [1-4]. In addition, this subgroup is the most common cause of the "toxic Strep syndrome" 
in immunocompromised, neutropenic hosts [5-8]. Given worldwide trends in penicillin resistance and $\beta$-lactam MIC (minimum inhibitory concentration) "creeps" amongst $S$. mitis-oralis strains, the proportion of serious infections caused by $\beta$-lactam-resistant strains in this VGS subgroup is alarming [8-11]. Of note, S. mitis-oralis strains have a propensity toward vancomycin-tolerance, further limiting therapeutic options. Collectively, these antibiotic resistance scenarios have rendered daptomycin (DAP) as a plausible option for treating invasive infections with this emerging pathogen. Interestingly, Garcia-de-la-Maria et al. recently reported that $>25 \%$ of $S$. mitis-oralis clinical strains can rapidly develop high-level and durable DAP-resistance (DAP-R; MICs $>256 \mu \mathrm{g} / \mathrm{mL}$ ) following both in vitro and in vivo (experimental IE) exposures to this agent [12]. Such a rapid evolution of high-level DAP-R has not been commonly seen clinically in other Gram-positive endovascular pathogens, including S. aureus and enterococci [13,14]. Although DAP-R VGS infections have only recently begun to be reported [12-14], the widespread use of DAP (both prophylactically and therapeutically) virtually assures that this scenario will occur more frequently with VGS. In addition, baseline DAP MICs among clinical S. mitis-oralis isolates are 2-10-fold higher than all other VGS subgroups [12].

DAP-R in S. mitis-oralis is based on unique mechanisms not seen in DAP-R S. aureus and enterococci. Thus, prior DAP-R S. mitis-oralis strains displayed an essentially complete disappearance of two key cell membrane (CM) phospholipids (PLs), phosphatidylglycerol (PG), and cardiolipin (CL), with the accumulation of phosphatidic acid (PA), the precursor PL in the CL biosynthetic pathway [15]. Of note, whole genome sequencing of several prototypic, in vitro passage-derived, isogenic DAP-S versus DAP-R S. mitis-oralis strain-pairs confirmed the presence of non-synonymous mutations within the key gene, $c d s A$, which encodes the first committed enzymatic step in the principal CL synthetic pathway (Figure S1) [15-17]. In contrast, in DAP-R S. mitis-oralis variants derived from DAP passage within a simulated ex vivo IE model, the above PG-CL disappearance signature was not consistently observed [18], and it was not associated with SNPs in either pgsA (also critical PL in the CL biosynthetic pathway) or $c d s A$. Of particular interest, some DAP-R S. mitis-oralis strains, particularly those harboring mutations in $c d s A$, show a cell-selective hyperaccumulation of DAP within only certain cocci within a chain, suggesting a form of "altruism" in which one cell subpopulation is 'protecting' the overall population [15]. However, this DAP hyperaccumulation phenotype is not uniformly found in all DAP-R strains. Collectively, these findings raised the possibility that the mechanism(s) of DAP-R among distinct $S$. mitis-oralis strains may co-depend on strain-specificities, as well as exposure scenarios (e.g., in vitro versus in simulated vegetations ex vivo versus within in vivo IE lesions).

The objective of this current study was to elucidate the hypothesis that individual S. mitis-oralis strains may not be "pre-programmed" in their adaptive pathways in terms of the emergence of DAP-R. Hence, such isolates may evoke and employ unique and multifactorial mechanisms of adaptations to DAP exposure in order to circumvent its bactericidal effects. Therefore, we investigated an additional, well-known S. mitis-oralis prototype strain (SF100) [19] isolated from an IE patient following its in vitro passage in DAP [20]. This investigation catalogued key and relevant genotypic and phenotypic perturbations related to the evolution of DAP-R in this strain to understand further the differential adaptation of DAP-R in this strain. This work was presented in-part at ASM Microbe Meeting; New Orleans, LA, USA; June 2017 [20].

\section{Methods}

\subsection{S. mitis-oralis Strains}

The DAP-S parental SF100 S. mitis-oralis strain used in this study was identified by MALDI-TOF, and has a DAP MIC of $1 \mu \mathrm{g} / \mathrm{mL}$ [20]). It is a clinical bloodstream isolate from a patient with IE without prior DAP exposures [19]; moreover, it is highly capable of inducing experimental IE [20]. In order to generate DAP-R derivatives, SF100 was subjected to a $20 \mathrm{~d}$ serial in vitro passage using concentrations of DAP $(20 \mu \mathrm{g} / \mathrm{mL})$ as described previously [20]. The DAP-R derivative variant was re-passaged in antibiotic-free brain heart infusion (BHI) media for five days to confirm the stability of the DAP-R 
phenotype [20]. MICs for the serial passage isolates were determined by microbroth dilution, as well as E test assays [15].

\subsection{Phospholipid (PL) Profiling}

Briefly, late stationary phase cells were used to extract the PLs from SF100 and its DAP-R variants, as described previously $[15,17,18]$. The major PLs (i.e., CL, PG, and PA, as well as a prominent glycolipid [GL] seen in all VGS) were separated by two-dimensional thin-layer chromatography (2D-TLC) (Silica 60 F254 HPTLC plates [Merck]). Identification of all the PL spots on the TLC plates was confirmed by comparison to known standards following exposure to iodine vapors and spraying with $\mathrm{CuSO}_{4}(100 \mathrm{mg} / \mathrm{mL})$ containing $8 \%$ phosphoric acid $(\mathrm{v} / \mathrm{v})$ and heated at $180{ }^{\circ} \mathrm{C}[15,17,18]$. The quantification of individual PLs removed directly from TLC plates was performed by digesting with $70 \%$ perchloric acid $(0.3 \mathrm{~mL})$ at $180{ }^{\circ} \mathrm{C}$ for $3 \mathrm{~h}$. The individual PLs were quantified by measuring the optical density at $660 \mathrm{~nm}$ using spectrophotometry as described before $[15,17,18]$. The PL results are expressed as their individual mean proportions $( \pm \mathrm{SD})$, employing at least three independent experiments performed on different days.

\subsection{Localization}

The fluorescent dye 10- $\mathrm{N}$-nonyl arcidine orange (NAO) was used to assess the localization of $\mathrm{CM}$ anionic PL-rich microdomains by previously described techniques [15]. Briefly, NAO preferentially binds to anionic PL-enriched microdomains (predominantly $\mathrm{CL}$ ) in the CM. For fluorescence microscopy, S. mitis-oralis cells were grown in TSB (Tryptic Soy Broth) to an exponential phase (A600 of approximately $0.3)$. Then, NAO was added to the growth medium to a final concentration of $1 \mu \mathrm{M}$, which did not inhibit the growth of S. mitis-oralis strains. Samples were stained for $3.5 \mathrm{~h}$ at $37^{\circ} \mathrm{C}$ with gentle shaking in the dark (because of light sensitivity). Study strains were washed three times with $0.9 \%$ saline post-incubation and immediately immobilized on a coverslip treated with poly-L-lysine (Sigma-Aldrich). Fluorescence imaging and phase-contrast imaging were carried out on an Olympus IX71 microscope with a PlanApo N 100-objective. Green fluorescence from NAO (representing anionic PLs) was detected by using a standard fluorescein isothiocyanate (FITC) filter (excitation at $490 \mathrm{~nm}$ and emission at $528 \mathrm{~nm}$ ). Image acquisition was performed using the SlideBook 5.0 software package. Three independent experiments were performed for each strain on different days.

\subsection{DAP Binding Assays}

Bodipy-DAP (BDP-DAP) was used to measure the interaction of DAP with the parental and DAP-R variants, as outlined before $[15,17]$. In brief, all study strains were grown in BHI broth at $37^{\circ} \mathrm{C}$, pelleted, and incubated with BDP-DAP (concentrations 4 and $64 \mu \mathrm{g} / \mathrm{mL}$ ) in LB (Luria-Bertani) broth supplemented with $\mathrm{Ca}^{2+}$ at $50 \mathrm{mg} /$ liter) for $15 \mathrm{~min}$ in the dark. After staining, excess BDP-DAP was removed by washing-centrifugation, the pellet was washed twice in LB broth, and then, it was immobilized on a coverslip pretreated with poly-L-lysine (Sigma-Aldrich St. Louis, MO, USA). Bacterial cells were seen with an Olympus IX71 fluorescence microscope equipped with PlanApo N 100× objective. Fluorescence measurements were carried out by using a standard FITC filter. The fluorescence intensity was quantitated and normalized to the protein concentration of the samples to assess the relative amount of BDP-DAP bound to the S. mitis-oralis isolates, as detailed elsewhere [15,17]. Protein concentrations above were measured by using standard bicinchoninic acid (BCA) protein assay kit (Thermo Scientific, West Hills, CA, USA) as instructed by the manufacturer described before [21,22]

\subsection{Surface Charge}

The relative positive surface charge of SF100 and its DAP-R variants was measured by using the cytochrome C (cyt C) binding assay following a spectrophotometric method $[15,17,18]$. Briefly, strains were grown in BHI broth, washed with $20 \mathrm{mM}$ MOPS (3-morpholinopropane-1-sulfonic acid) buffer $(\mathrm{pH} 7.0)$, and resuspended in the same buffer at $\mathrm{OD}_{578}=1.0$ Cells were incubated with $0.5 \mathrm{mg} / \mathrm{mL}$ 
cyt $C$ for $10 \mathrm{~min}$, and the amount of cyt $C$ (a highly positively charged molecule) remaining in the supernatant after 15 min exposure was measured spectrophotometrically at $\mathrm{OD}_{530} \mathrm{~nm}$. The greater the amount of cyt $C$ detected in the supernatant (i.e., more unbound cyt $C$ ) equates to a relatively increased positive surface charge $[15,17,18]$. The quantified data represented the means $( \pm \mathrm{SD})$ of the amount of cyt $C$ detected in the supernatant from a minimum of three independent experiments.

\subsection{Fluidity}

Fluidity was determined by using the fluorescent probe 1,6-diphenyl-1,3,5-hexatriene (DPH; excitation and emission wavelengths are $360 \mathrm{~nm}$ and $426 \mathrm{~nm}$, respectively). The protocol of DPH incorporation into the $\mathrm{CM}$ has been described in detail previously $[15,17,18]$. Spectrofluorimetry was used to quantify fluorescence polarization and define the polarization index (PI) value as done before (BioTek SFM 25) [15]. An inverse correlation exists between PI value and fluidity (i.e., a lower PI value equates to a higher extent of CM fluidity) $[15,17,18]$. A minimum of three independent experiments were conducted for each isolate on different days.

\subsection{Whole Genome Sequencing}

Genomic DNA from SF100 WT and a DAP-resistant variant isolated on day 20 of passage (strain D20) from the VGS strain-set was isolated by cetyltrimethylammonium bromide (CTAB) extraction as described previously $[15,20]$. Genomic DNA was further purified for sequencing using a PureLink Genome DNA Minikit (Invitrogen Waltham, MA, USA) according to the manufacturer's instructions $[15,20]$. PacBio library construction, sequencing, and annotation were conducted by using standard methods $[15,20]$. The generation of genome assemblies and subsequent identification of single nucleotide polymorphisms (SNPs) and insertion-deletion mutations (indels) were performed as detailed elsewhere $[15,20]$. All mutations identified by whole genome sequencing were confirmed by PCR and Sanger sequencing.

\subsection{Statistics}

The two-tailed Students $t$-test was utilized for statistical analysis of all quantitative data. $p$ values of $\leq 0.05$ were considered "significant".

\section{Results}

\subsection{Generation of DAP-R S. mitis-oralis Strain SF100}

Following a $20 \mathrm{~d}$ serial passage in DAP, the DAP MIC of SF100 progressively rose to $>256 \mu \mathrm{g} / \mathrm{mL}$ (Table 1) [20]. This phenotype was stable on the passage of this DAP-R variant in antibiotic-free media.

Table 1. List of S. mitis-oralis strains and their daptomycin (DAP) MICs.

\begin{tabular}{ccc}
\hline Strains & Relevant Characteristics & DAP MIC $(\mu \mathrm{g} / \mathbf{m L})$ \\
\hline SF100 & Endocarditis isolate from patient & 1 \\
D10 & DAP-R derivative produced by in vitro passage & 64 \\
D15 & DAP-R derivative produced by in vitro passage & $>256$ \\
D20 & DAP-R derivative produced by in vitro passage & $>256$ \\
\hline
\end{tabular}

\subsection{PL Content of DAP-R S. mitis-oralis Strains}

As noted for other S. mitis-oralis strains [15,17,18], 2D-TLC of parental DAP-S strain SF100 revealed four major lipid species: three PLs (PG, CL, and PA) and a large glycolipid (GL) spot. The day 20 (D20) DAP-R passage variant exhibited a significant reduction of CL content as compared to the SF100 parental strain (Table 2). Furthermore, the amount of PG was not altered significantly in the SF100 D20 strain versus the DAP-S parental strain. The quantitative PL assays paralleled the qualitative 2D-TLC 
plate assays above (Figure S2). Of interest, the current dataset was somewhat distinct from our prior study in which a prototypic DAP-R S. mitis-oralis strain showed a virtual absence of both PG and CL in the CM [15].

Table 2. Phospholipids (PL) content of study strains. CL: cardiolipin, PA: phosphatidic acid, PG: phosphatidylglycerol.

\begin{tabular}{cccc}
\hline \multirow{2}{*}{ Strains } & \multicolumn{3}{c}{ CM PL Composition (\% of Total PL [Mean \pm SD]) } \\
\cline { 2 - 4 } & PG & CL & PA \\
\hline SF100 & $26 \pm 6$ & $52 \pm 9$ & $21 \pm 10$ \\
D10 & $24 \pm 6$ & $40 \pm 14^{*}$ & $36 \pm 14^{*}$ \\
D15 & $28 \pm 9$ & $32 \pm 15^{*}$ & $40 \pm 14^{*}$ \\
D20 & $34 \pm 13$ & $25 \pm 11^{*}$ & $41 \pm 17^{*}$ \\
\hline
\end{tabular}

${ }^{*} p$ value $<0.05$ vs. SF100 parental strain.

\subsection{Distribution of Anionic PL Microdomains}

The parental SF100 DAP-S strain (Figure 1A) exhibited anionic PL microdomains mainly located at the septa similar to other DAP-S Gram-positive bacteria [15]. In addition, non-septal discrete fluorescent foci were also observed, which is reminiscent of the ExPortal found in S. pyogenes [23,24]. However, in contrast to prior studies [15], septal anionic PL staining was lost in DAP-R derivatives of SF100 (Figure 1B-D); these findings suggested a redistribution of anionic PL microdomains (e.g., CL) away from the septum; this redistribution may well play an important role in DAP-R in S. mitis-oralis strains (mirroring similar data in DAP-R E. faecalis) [22].

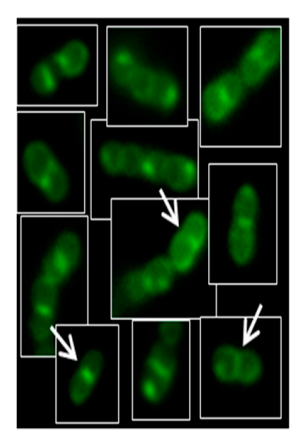

(A)

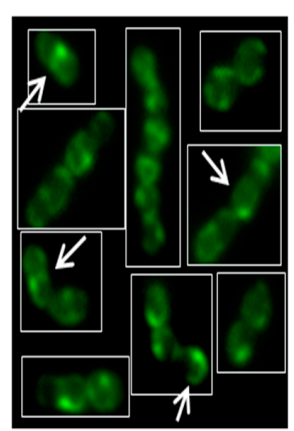

(B)

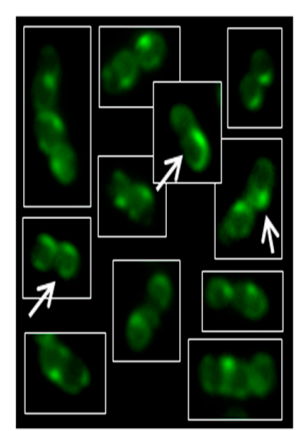

(C)

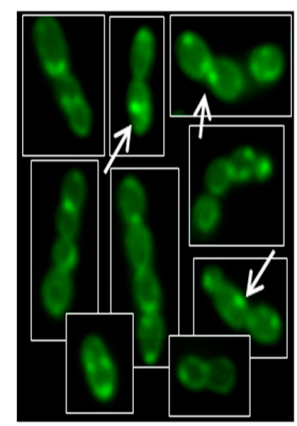

(D)

Figure 1. S. mitis-oralis DAP-S SF100 and its DAP-R variants at days 10, 15, 20 post-in vitro DAP passage. Panel A: arrows indicate anionic PL localization at the division septum; Panel B-D: arrows indicate redistribution of anionic PLs away from division septum in DAP-R derivatives (D10, D15, and D20).

\subsection{Cell Surface Positive Charge}

It has been well-chronicled that many DAP-R S. aureus, enterococci, and some prior S. mitis-oralis strains exhibit increases in relative positive surface charge versus their respective DAP-S parental strains $[15,17,18,21,23]$. This DAP-R adaptation has been proposed to generate a "charge-repulsive milieu" that contributes to a reduced ability of calcium-DAP to bind to target CMs to initiate its bactericidal effects. Surprisingly, and in contradistinction to a previous study [15], as SF100 evolved stable DAP-R upon in vitro passage, its surface became substantially less positively charged (Table 3).

\subsection{DAP CM Binding Profiles}

As opposed to a prior study [15], in comparing the parental SF100 strain with DAP-R SF100 D20, no quantitative differences in overall DAP binding were detected at either DAP exposure concentration (Figure 2). 
Table 3. Positive surface charge and cell membrane (CM) fluidity of S. mitis-oralis SF100 and its DAP-R variants at days 10,15 , and 20 post-in vitro DAP passage.

\begin{tabular}{ccc}
\hline S. mitis-oralis Strains & $\begin{array}{c}\text { Surface Charge } \\
\text { (\% of cyt C in Supernatant) }\end{array}$ & $\begin{array}{c}\text { CM Fluidity } \\
\text { (PI Value) }\end{array}$ \\
\hline SF100 & $94 \pm 13$ & $0.399 \pm 0.0$ \\
D10 & $46 \pm 17 *$ & $0.350 \pm 0.1$ \\
D15 & $75 \pm 24$ & $0.388 \pm 0.1$ \\
D20 & $62 \pm 16 *$ & $0.298 \pm 0.0 *$ \\
\hline
\end{tabular}

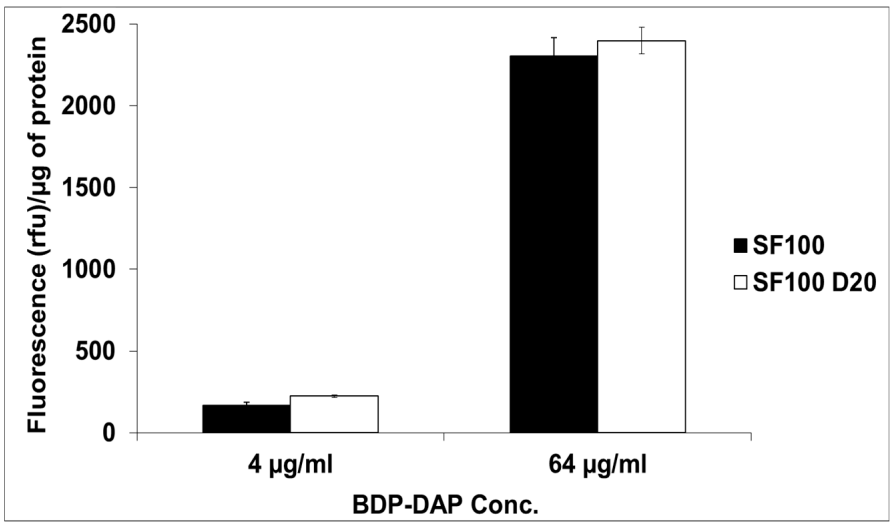

Figure 2. Bodipy-DAP (BDP-DAP) Binding DAP-S SF100 parental versus DAP-R SF100 D20 S. mitis-oralis.

\subsection{Fluidity}

It is well known that the relative state of the CM "order" has a profound impact on the ability of calcium-DAP and other cationic peptides to interact with the target CM. Interestingly, enhancements in fluidity were, indeed, observed in DAP-R variants; of interest, this phenotype was somewhat delayed during the evolution of DAP-R, becoming evident only in the D20 DAP-R passage variant (Table 3).

\subsection{Whole-Genome Sequencing (WGS)}

WGS of SF100D20 versus the SF100 strain pair revealed a relatively limited number of non-synonymous SNPs and deletions emerging in the DAP-R SF100 D20 passage variant. Of note, these mutations were broadly grouped into those impacting (1) lipid metabolism; (2) cell wall and cell surface homeostasis; or (3) ion transport and the maintenance of CM potential. As underscored above, each of these latter phenotypes are plausibly critical in the interaction of DAP with target CMs $[15,17,18,21,22]$.

As compared with its parent strain, the DAP-R variant, SF100 D20, contains 13 SNPs and 2 deletions, involving a total of 17 ORFs After eliminating genes of unknown function, as well as those with no apparent functional linkage to DAP-R, we identified four candidate genes containing non-synonymous SNPs that could likely be linked to DAP-R (Table 4). Similar to a previous study [17], an SNP in $p g s A$ (ORF853) was identified in the SF100 D20 strain; this enzyme catalyzes an important step in CL biosynthesis (Figure S1). In addition, an SNP was found in ORF1433, which is predicted to encode an acetyl-coA-acetyl transferase; this is a key enzyme in the mevalonate pathway, which, in turn, is important for fatty acid metabolism and CM order maintenance, especially under stress conditions (e.g., 'cold shock') [25]. ORF1632 (rpoC) encodes the $\beta^{\prime}$ subunit of RNA polymerase. Mutations in this protein have been linked to DAP-R [25]. Of interest, RpoBC in S. aureus can repress dlt expression, the latter being a key regulator of surface charge maintenance [26]. Thus, an SNP in rpoC could impact the surface positive charge and potentially perturb calcium-DAP surface binding. 
SNPs were also found in ORF1590, encoding an alanine dehydrogenase (ald) homologue. Of note, mutations in these latter two genes in S. aureus have each been associated with cationic antimicrobial peptide resistance $[27,28]$.

Table 4. Potential candidate genes for mediating DAP-R in S. mitis-oralis SF100 D20.

\begin{tabular}{ccccc}
\hline ORF $^{*}$ & NSNP & AA Change & Name & Biologic Role and Comments \\
\hline 674 & G446A & T->I & $\begin{array}{c}\text { alanine dehydrogenase }(a l d) \\
\text { homologue }\end{array}$ & $\begin{array}{c}\text { Cellular energy metabolism; SCV } \\
\text { (small colony variant) phenotype }\end{array}$ \\
\hline 1418 & C194T & G->E & $\begin{array}{c}\text { CDP-diacylglycerol-glycerol-3- } \\
\text { phosphate 3-phosphatidyl- } \\
\text { transferase }(p g s A)\end{array}$ & CM phospholipid synthesis \\
\hline 1433 & G631T & P->T & $\begin{array}{c}\text { acetyl-coA acetyl transferase } \\
(t h l A)\end{array}$ & mevalonate pathway, integrity of \\
CM32 & G1874A & T->I & RNA polymerase $\beta^{\prime}$ subunit \\
$(r p o C)$ & $\begin{array}{c}\text { Interacts with } \text { dlt operon; involved in } \\
\text { maintenance of surface charge }\end{array}$ \\
\hline
\end{tabular}

* Open reading frame (ORF) in SF100; ** non-synonymous single nucleotide polymorphism.

\section{Discussion}

The current study with in vitro-derived DAP-R variants emerging from strain SF100 was compelling for a number of reasons. For example, several of the genetic mutations we observed evolving in the SF100 DAP-R variants have been previously linked to DAP-R in other S. mitis-oralis (e.g., pgsA) [17]. Moreover, other documented mutations we observed were in genes involved in metabolic pathways important for CM biogenesis, integrity, or transmembrane potential maintenance, making them plausible candidates for a role in DAP-R. In addition, several of these genes are important in terms of other metabolic parameters that are likely involved in DAP-R (e.g., fatty acid metabolism; $\mathrm{Na}^{+} / \mathrm{H}^{+}$antiporters).

Of note, we did not observe non-synonymous mutations in the $c d s A$ gene in SF100 D20 DAP-R variants as documented in other DAP-R S. miti-oralis [15]. However, it should be emphasized that (1) we did identify SNPs in the $p g s A$ gene that catalyze the production of PG, the PL that eventually dimerizes to form CL; and (2) many additional regulatory and metabolic pathways can impact CL synthesis, other than the primary pathway shown in Figure S1 [15]. In this context, WGS of DAP-R in $S$. aureus and B. subtilis $[25,26]$ has also identified mutations in a number of genes responsible for PL biosynthesis, including $p g s A$. Thus, it is likely that the mutations identified above by WGS synergize to yield the DAP-R phenotype in SF100.

To assess the phenotypic correlation of mutations in $p g s A$ that were found on WGS, 2D TLC and NAO microscopy were performed to quantify proportional PL content, as well as the localization and distribution of anionic PLs, respectively. We recently documented a unique phenotype in other DAP-R S. mitis-oralis strains, i.e., an apparent complete disappearance of PG and CL, with the accumulation of the PL precursor, PA [15]. Since negatively charged PG is essential for interaction with, and the oligomerization of DAP within target CMs [29], this phenotype was logically linked to DAP-R. In addition, negatively charged PLs (such as PG and CL) are crucial in DAP interactions within the cell envelope at its major site of activity, the septal division plane. In addition, mutations in $c d s A$ and $c l s$ (leading to alterations in CL content) in DAP-R S. mitis-oralis and enterococci have been associated with the diversion and mislocalization of DAP away from the septum [15,22]. Moreover, the combined "microdomains" of PG and CL are known to act as a negatively charged CM "docking site" for DAP and other cationic molecules after initial interaction with and penetration of the CM.

In contrast to these prior investigations [15], we did not observe a "PG-CL disappearance phenotype" among DAP-R variants derived from the in vitro DAP passaging of strain SF100. Of note, DAP-R strains exhibited a similar pattern of PG content versus its DAP-S SF100 parental strain; however, a significant reduction of CL content was noted among the DAP-R variants. It is possible that 
the mutation in $p g s A$ noted above in the DAP-R strain impacted CL content by reducing the amount of PG available for ultimate dimerization into CL.

In addition, NAO staining assays indicated that anionic PL-enriched microdomains were localized at the septum in the DAP-S parental strain as expected. However, in the DAP-R variant, anionic PLs were diverted away from the division septum, likely contributing to DAP-R, as seen in DAP-R enterococci [22].

Another notable feature of prior S. mitis-oralis and other DAP-R organisms is increased positive surface charge $[15,17,18]$. In contrast, in the current study, the net surface charge of the DAP-R variant became less positive during the evolution of DAP-R in vitro. These data underscore that a "charge-repulsive cell surface" is only one of a number of factors that can potentially account for the DAP-R phenotype. In addition, it is theoretically possible that a more negatively charged surface can "trap" more calcium-DAP and prevent it from interfacing with the CM PLs required for its ultimate mechanisms of action.

The DAP binding assays for the DAP-R SF100 variant yielded data with very different outcomes as compared to other DAP-R S. mitis-oralis derivatives [15]. Thus, prior studies showed that DAP binding was surprisingly increased overall in DAP-R variants that harbor mutations in $c d s A$, which is associated with the selective hyperaccumulation of DAP in a minority of cocci within individual VGS chains. This supported the notion that in those DAP-R strains, such DAP-hyperaccumulating cells were, in essence, "protecting" the majority of population from exposure to DAP (an example of "altruism") [15]. In contrast, we found no difference in the quantitative amount of DAP binding when comparing our parent versus DAP-R S. mitis-oralis variant.

Alterations in CM fluidity have been linked to DAP-R and cross-resistance to other cationic antimicrobial peptides among other DAP-R Gram-positive pathogens [13,15,17,30-32]. It is believed that there exists a fluidity optimum for the interaction of specific cationic antimicrobial peptides (such as calcium-DAP) with target bacterial CMs. The SF100 DAP-R strains exhibited significant increases in CM fluidity versus its DAP-S parental strain, similar to previous observations, suggesting that this biophysical metric may be contributing to DAP-R [15].

\section{Conclusions}

It is likely that S. mitis-oralis can develop DAP-R via several mechanisms, as has been shown in studies of DAP-R in S. aureus and enterococci [30-32]. However, the data presented here, in combination with previous work $[15-17,33]$ indicate that altered membrane composition and some other metabolic modifications [34] may be a conserved mechanism for DAP-R. Undoubtedly, specific S. mitis-oralis strains are not "pre-programmed" to develop DAP-R by any single pathway.

Supplementary Materials: The following are available online at http://www.mdpi.com/2079-6382/9/8/520/s1, Figure S1: Major steps in the CL biosynthetic pathways; Figure S2. Major CM PLs of S. mitis-oralis strains. Proportions of PG, CL, and PA in CM of SF100 and its DAP-R derivative SF100 D20 were determined by 2D thin-layer chromatography. GL is a major CM glycolipid found in all VGS.

Author Contributions: Conceptualization, N.N.M. and A.S.B.; methodology, N.N.M., T.T.T., R.T.; formal analysis, N.N.M. A.S.B., T.T.T., C.A.A., P.M.S.; investigation, N.N.M. A.S.B., T.T.T., C.A.A., R.S., P.M.S.; resources, N.N.M., A.S.B., C.A.A., P.M.S.; data curation, N.N.M., A.S.B., T.T.T., C.A.A., R.S. P.M.S.; writing-original draft preparation, N.N.M., A.S.B.; writing-review and editing, N.N.M., A.S.B., T.T.T., C.A.A., R.S., P.M.S.; visualization, N.N.M., T.T.T.; supervision, N.N.M., A.S.B.; project administration, N.N.M., A.S.B.; funding N.N.M., A.S.B. All authors have read and agreed to the published version of the manuscript.

Funding: Cubist Pharmaceuticals (21272-01) and The Lundquist Institute-Harbor UCLA Intramural Research Grant 531604-01-01 to N.N.M.; National Institutes of Health (1R01130056-01 to A.S.B.; NIAID K24 AI121296, R01 AI134637 and R01 AI148342 to C.A.A.; K08 AI113317 to T.T.T., and R01 AI41513 to P.M.S.

Acknowledgments: We are thankful to Danya Alvarez for technical assistance. We also thank Luke Tallon and Suvarna Nadendla at the University of Maryland Institute for Genome Sciences for their help with whole genome sequencing and analysis.

Conflicts of Interest: The authors declare no conflict of interest. 


\section{References}

1. Holland, T.L.; Bayer, A.S.; Fowler, V.G. Endocarditis and Intravascular Infections. In Principles and Practices of Infectious Diseases; Mandel, G.L., Bennett, J.E., Dolin, R., Blaser, M.J., Eds.; Elsevier: Amsterdam, The Netherlands, 2019; Chapter 80.

2. Ahmed, R.; Hassall, T.; Morland, B.; Gray, J. Viridans streptococcus bacteremia in children on chemotherapy for cancer: An underestimated problem. Pediatr. Hematol. Oncol. 2003, 20, 439-444. [CrossRef] [PubMed]

3. Husain, E.; Whitehead, S.; Castell, A.; Thomas, E.E.; Speert, D.P. Viridans streptococci bacteremia in children with malignancy: Relevance of species identification and penicillin susceptibility. Pediatr. Infect. Dis. J. 2005, 24, 563-566. [CrossRef] [PubMed]

4. Marron, A.; Carratala, J.; Gonzalez-Barca, E.; Fernandez-Sevilla, A.; Alcaide, F.; Gudiol, F. Serious complications of bacteremia caused by Viridans streptococci in neutropenic patients with cancer. Clin. Infect. Dis. 2000, 31, 1126-1130. [CrossRef]

5. Huang, W.T.; Chang, L.Y.; Hsueh, P.R.; Lu, C.Y.; Shao, P.L.; Huang, F.Y.; Lee, P.I.; Chen, C.M.; Lee, C.Y.; Huang, L.M. Clinical features and complications of viridans streptococci bloodstream infection in pediatric hemato-oncology patients. J. Microbiol. Immunol. Infect. 2007, 40, 349-354.

6. Shelburne, S.A.; Sahasrabhojane, P.; Saldana, M.; Hui, Y.; Xiaoping, S.; Horstmann, N.; Thompson, E.; Flores, A.R. Streptococcus mitis strains causing severe clinical disease in cancer patients. Emerg. Infect. Dis. 2014, 20, 762-771. [CrossRef]

7. Freifeld, A.G.; Razonable, R.R. Viridans group streptococci in febrile neutropenic cancer patients: What should we fear? Clin. Infect. Dis. 2014, 59, 231-233. [CrossRef]

8. Prabhu, R.M.; Piper, K.E.; Baddour, L.M.; Steckelberg, J.M.; Wilson, W.R.; Patel, R. Antimicrobial susceptibility patterns among viridans group streptococci isolates from infective endocarditis patients from 1971-1986 and 1994-2002. Antimicrob. Agents Chemother. 2004, 48, 4463-4465. [CrossRef]

9. Shelburne, S.A.; Lasky, R.E.; Sahasrabhojane, P.; Tarrand, J.T.; Rolston, K.V.I. Development and validation of a clinical model to predict the presence of $\beta$-lactam resistance in viridians group streptococci causing bacteremia in neutropenic cancer patients. Clin. Infect. Dis. 2014, 59, 223-230. [CrossRef]

10. Ron-Bin, H.; Lin, F.-Y. Effect of penicillin resistance on presentation and outcome of nonenterococcal streptococcal infective endocarditis. Cardiology 2006, 105, 234-239.

11. Sabella, C.; Murphy, D.; Drummond-Webb, J. Endocarditis due to Streptococcus mitis with high-level resistance to penicillin and ceftriaxone. JAMA 2001, 285, 2195. [CrossRef]

12. Garcia-de-la-Maria, C.; Pericas, J.M.; del Rio, A.; Castañeda, X.; Vila-Farrés, X.; Armero, Y.; Espinal, P.A.; Cervera, C.; Soy, D.; Falces, C.; et al. Early In Vitro and In Vivo development of high-level daptomycin resistance is common in mitis group of streptococci after exposure to daptomycin. Antimicrob. Agents Chemother. 2013, 57, 2319-2325. [CrossRef] [PubMed]

13. Bayer, A.S.; Schneider, T.; Sahl, H.-G. Mechanisms of daptomycin resistance in Staphylococcus aureus: Role of the cell membrane and cell wall. Ann. NY Acad. Sci. 2013, 1277, 139-158. [CrossRef] [PubMed]

14. Kaatz, G.W.; Lundstrom, T.S.; Seo, S.M. Mechanisms of daptomycin resistance in Staphylococcus aureus. Int. J. Antimicrob. Agents. 2006, 28, 280-287. [CrossRef] [PubMed]

15. Mishra, N.N.; Tran, T.T.; Seepersaud, R.; Garcia-de-la-Maria, C.; Faull, K.; Yoon, A.; Miro, J.M.; Rybak, M.J.; Bayer, A.S.; Arias, C.A.; et al. Perturbations of phosphatidate cytidylyltransferase (CdsA) mediate daptomycin resistance in Streptococcus mitis by a novel mechasnism. Antimicrob. Agents Chemother. 2017. [CrossRef]

16. Adams, H.M.; Joyce, L.R.; Guan, Z.; Akins, R.L.; Palmer, K.L. Streptococcus mitis and S. oralis lack a requirement for CdsA, the enzyme required for synthesis of major membrane phospholipids in bacteria. Antimicrob. Agents Chemother. 2017. [CrossRef]

17. Tran, T.T.; Mishra, N.N.; Seepersaud, R.; Diaz, L.; Rios, R.; Dinh, A.Q.; Garcia-de-la-Maria, C.; Rybak, M.J.; Miro, J.M.; Shelburne, S.; et al. Mutations in $c d s A$ and $p g s A$ correlate with daptomycin resistance in Streptococcus mitis and S. oralis. Antimicrob. Agents Chemother. 2018, 63, e01531-18. [CrossRef]

18. Kebriaei, R.; Rice, S.A.; Stamper, K.C.; Seepersaud, R.; Garcia-de-la-Maria, C.; Mishra, N.N.; Miro, J.M.; Arias, C.A.; Tran, T.T.; Sullam, P.M.; et al. Daptomycin dose-ranging evaluation with single-dose versus multidose ceftriaxone combinations against Streptococcus mitis/oralis in an Ex Vivo simulated endocarditis vegetation model. Antimicrob. Agents Chemother. 2019, 63, e00386-19. [CrossRef] 
19. Seo, H.S.; Sullam, P.M. Characterization of the fibrinogen-binding domain of bacteriophage lysin from Streptococcus mitis. Infect. Immun. 2011, 79, 3518-3526. [CrossRef]

20. Mishra, N.N.; Seepersaud, R.; Tran, T.T.; Shelburne, S.; Miro, J.M.; Rybak, M.J.; Arias, C.A.; Sullam, P.; Bayer, A.S. Phenotypic and Genotypic Characteristics of In Vitro-Selected High Level Daptomycin-Resistant (DAP-R) Strains of Clinically Derived Streptococcus mitis-oralis. In Proceedings of the ASM Microbe, New Orleans, LA, USA, 1-5 June 2017.

21. Bogdanov, M.; Dowhan, W. Phosphatidylethanolamine is required for in vivo function of the membraneassociated lactose permease of Escherichia coli. J. Biol. Chem. 1995, 270, 732-739. [CrossRef]

22. Tran, T.T.; Panesso, D.; Mishra, N.N.; Mileykovskaya, E.; Guan, Z.; Munita, J.M.; Reyes, J.; Diaz, L.; Weinstock, G.M.; Murray, B.E.; et al. Daptomycin-resistant Enterococcus faecalis diverts the antibiotic molecule from the division septum. mBio 2013, 23, e00281-13. [CrossRef]

23. Rosch, J.W.; Hsu, F.F.; Caparon, M.G. Anionic lipids enriched at the ExPortal of Streptococcus pyogenes. J. Bacteriol. 2017, 189, 801-806. [CrossRef] [PubMed]

24. Vega, L.A.; Caparon, M.G. Cationic antimicrobial peptides disrupt the Streptococcus pyogenes ExPortal. Mol. Microbiol. 2012, 85, 1119-1132. [CrossRef] [PubMed]

25. Klein, W.; Weber, M.H.; Marahiel, M.A. Cold shock response of Bacillus subtilis: Isoleucine-dependent switch in the fatty acid branching pattern for membrane adaptation to low temperatures. J. Bacteriol. 1999, 181, 5341-5349. [CrossRef] [PubMed]

26. Friedman, L.; Alder, J.D.; Silverman, J.A. Genetic changes that correlate with reduced susceptibility to daptomycin in Staphylococcus aureus. Antimicrob. Agents Chemother. 2006, 50, 2137-2145. [CrossRef]

27. Bayer, A.S.; McNamara, P.; Yeaman, M.R.; Lucindo, N.; Jones, T.; Cheung, A.L.; Sahl, H.-G.; Proctor, R.A. Transposon disruption of the complex I NADH oxidoreductase gene (snoD) in Staphylococcus aureus is associated with reduced susceptibility to the microbicidal activity of thrombin-induced platelet microbicidal protein 1. J. Bacteriol. 2006, 188, 211-222. [CrossRef]

28. Kohler, C.; Proctor, R.A.; Bayer, A.S.; Yeaman, M.R.; Lalk, M.; Engelmann, S.; Mishra, N.N. Proteomic and membrane lipid analysis of a snoD mutant of Staphylococcus aureus: Insights into host defense peptide resistance. Antibiotics (Basel) 2019, 8, 169. [CrossRef]

29. Muraih, J.K.; Pearson, A.; Silverman, J.; Palmer, M. Oligomerization of daptomycin on membranes. Biochim. Biophys. Acta (BBA) Biomembr. 2011, 1808, 1154-1160. [CrossRef]

30. Mishra, N.N.; Yang, S.-J.; Chen, L.; Muller, C.; Saleh-Mghir, A.; Kuhn, S.; Peschel, A.; Yeaman, M.R.; Nast, C.C.; Kreiswirth, B.N. Emergence of daptomycin resistance in daptomycin-naïve rabbits with methicillin-resistant Staphylococcus aureus prosthetic joint infection is associated with resistance to host defense cationic peptides and mprF polymorphisms. PLoS ONE 2013, 8, e71151. [CrossRef]

31. Mishra, N.N.; Bayer, A.S.; Tran, T.T.; Shamoo, Y.; Mileykovskaya, E.; Dowhan, W.; Guan, Z.; Arias, C.A. Daptomycin resistance in enterococci is associated with distinct alterations of cell membrane phospholipid and cell envelope characteristics. PLOS ONE 2012, 7, e43958. [CrossRef]

32. Mishra, N.N.; Rubio, A.; Nast, C.C.; Bayer, A.S. Differential adaptations of methicillin-resistant Staphylococcus aureus to serial in vitro passage in daptomycin: Evolution of daptomycin resistance and the role of membrane carotenoid content and fluidity. Intl. J. Microbiol. 2012, 2012, 68345. [CrossRef]

33. Adams, H.M.; Joyce, L.R.; Guan, Z.; Akins, R.L.; Palmer, K.L. Streptococcus mitis and Streptococcus oralis mutate an 'essential' gene upon exposure to daptomycin. FASEB J. 2018. [CrossRef]

34. Parrett, A.; Reed, J.M.; Gardner, S.G.; Mishra, N.N.; Bayer, A.S.; Powers, R.; Somerville, G.A. Metabolic changes associated with adaptive resistance to daptomycin in Streptococcus mitis-oralis. BMC Microbiol. 2020, 20, 162. [CrossRef] [PubMed]

(C) 2020 by the authors. Licensee MDPI, Basel, Switzerland. This article is an open access article distributed under the terms and conditions of the Creative Commons Attribution (CC BY) license (http://creativecommons.org/licenses/by/4.0/). 